\title{
Direct Mismatch Characterization of femto-Farad Capacitors
}

\author{
Hesham Omran, Rami T. ElAfandy, Muhammad Arsalan, Member, IEEE, and Khaled N. Salama, Senior \\ Member, IEEE
}

\begin{abstract}
Reducing the capacitance of programmable capacitor arrays, commonly used in analog integrated circuits, is necessary for low-energy applications. However, limited mismatch data is available for small capacitors. We report mismatch measurement for a $2 f F$ poly-insulator-poly (PIP) capacitor, which is the smallest reported PIP capacitor to the best of the authors' knowledge. Instead of using complicated custom onchip circuitry, direct mismatch measurement is demonstrated and verified using Monte Carlo Simulations and experimental measurements. Capacitive test structures composed of $9-b i t$ programmable capacitor arrays (PCAs) are implemented in a low-cost $0.35 \mu \mathrm{m}$ CMOS process. Measured data is compared to mismatch of large PIP capacitors, theoretical models, and recently published data. Measurement results indicate an estimated average relative standard deviation of $\mathbf{0 . 4 3 \%}$ for the $2 f F$ unit capacitor, which is better than the reported mismatch of metal-oxide-metal (MOM) fringing capacitors implemented in an advanced $32 \mathrm{~nm}$ CMOS process.
\end{abstract}

Index Terms-Capacitor mismatch, mismatch characterization, programmable capacitor array (PCA), energy-efficient circuits, analog-to-digital converter (ADC), capacitance-to-digital converter (CDC)

\section{INTRODUCTION}

$\mathbf{P}$ ROGRAMMABLE capacitor arrays (PCAs) are widely used in analog and mixed-signal circuit design, especially in data-converter circuits [1]-[5]. For instance, capacitive digital-to-analog converter (DAC) is an essential component of charge redistribution successive approximation register (SAR) analog-to-digital converter (ADC). The matching between the unit elements of the capacitive DAC is the primary factor affecting the linearity of the whole ADC [6]. On the other hand, reducing the power consumption of the capacitor array by reducing the unit capacitor to few femto-Farad or sub-femtoFarad ranges is essential to optimize the energy efficiency of the system, where $k T / C$ noise imposes the lower bound on the total capacitance [5]. These two requirements impose a trade-off in the design process because the matching becomes worse as the unit capacitor becomes smaller according to the well-known Pelgrom's model [7]. In addition to being essential for energy-efficient design, well-matched small unit capacitors also set the absolute resolution and the dynamic range of direct capacitance-to-digital converters (CDCs) that employ

Hesham Omran, Rami T. ElAfandy, and Khaled N. Salama are with the Electrical Engineering Program, King Abdullah University of Science and Technology (KAUST), Thuwal 23955-6900, Saudi Arabia (email: \{hesham.omran, rami.afandy, khaled.salama\}@kaust.edu.sa). Muhammad Arsalan is with the Production Technology Division of the Expec Advanced Research Center (Expec ARC), Saudi Aramco, Dhahran 31311, Saudi Arabia, (email: muhammad.arsalan@aramco.com). successive approximation technique [3], [4], and can be used as dummy capacitive sensors for CDC testing [8].

In spite of the importance of small matched capacitors, limited measurement data is available on their mismatch characteristics. Capacitor mismatch models available in semiconductor foundries design kits are based on mismatch measurements of large capacitive structures. Small capacitors either have mismatch models based on extrapolating measurements of large capacitors assuming Pelgrom's model, or are not available at all and have to be customly crafted as a layout parasitic element. Mismatch measurement of small capacitors using onchip circuitry is reported in [9], [10]. In [9], a test structure that uses differential ring oscillator is proposed to measure mismatch of small metal-oxide-metal (MOM) sandwich and fringing capacitors down to $8 f F$. However, the proposed technique suffers from several sources of errors due to resistor matching, parasitic capacitance, and frequency variation due to loading effects. In addition, mapping frequency measurement to capacitor mismatch data involves a complicated and errorprone process where component values are tuned in simulation till simulation results fit with measured data. The second technique reported in [10] employs an on-chip ADC to digitize the mismatch of MOM fringing capacitor as small as $0.45 \mathrm{fF}$. This technique provides direct digital output that contains mismatch information; however, complicated analog circuitry is required on-chip. This circuitry is customly designed and laid-out for a given technology; thus it consumes valuable design time and silicon area, and cannot be easily ported to a different technology. In [5], mismatch of $0.5 f F$ MOM capacitors was estimated based on linearity measurement of a SAR ADC. In addition to the disadvantages of [10], this indirect method is affected by other sources of error in the linearity measurement and in the ADC itself, which degrades the measurement accuracy.

In this brief, we report direct mismatch measurement of $2 f F$ poly-insulator-poly (PIP) capacitor, which is the smallest reported PIP capacitor to the best of the authors' knowledge. The feasibility of direct measurement of small capacitor mismatch is demonstrated; thus complicated and error-prone custom on-chip circuitry is avoided. A simple capacitor array is used as a test structure; thus the layout process can be easily automated and applied to different technologies. Measured results are compared with Pelgrom's model which is based on large capacitor mismatch measurement, showing that small capacitors will suffer from increased mismatch due to edge effects [7], [11]. Experimental measurements show that the relative variation of $2 f F$ PIP capacitors implemented in 


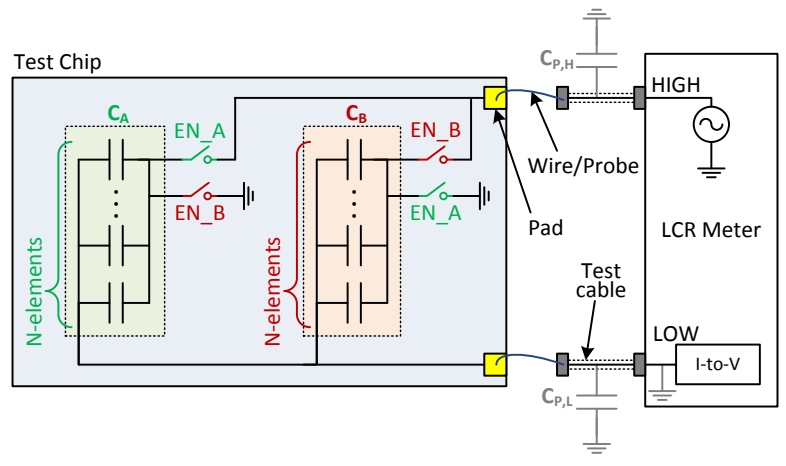

Figure 1. Schematic illustrating direct mismatch measurement between one pair of capacitors $\left(C_{A}\right.$ and $\left.C_{B}\right) . C_{P, H}$ and $C_{P, L}$ model parasitic capacitance at the HIGH and LOW terminals respectively.

$0.35 \mu \mathrm{m}$ low-cost CMOS technology has a standard deviation of $0.43 \%$, which is better than the mismatch of an equal MOM capacitor implemented in $32 \mathrm{~nm}$ process. Thus energyefficient capacitor arrays can be implemented in a low-cost process, though advanced nodes will remain the choice if higher integration density is required.

\section{Direct Mismatch Measurement}

A unit capacitor can be represented as a normally distributed random variable given by

$$
C=C_{\mu}+\Delta C
$$

where $C_{\mu}$ is the unit capacitor nominal value and $\Delta C$ is the deviation from the nominal value due to random process variations. The absolute standard deviation of the unit capacitor is given by $\sigma(C)=\sigma(\Delta C)$, while the relative standard deviation is $\sigma\left(\frac{\Delta C}{C_{\mu}}\right)$.

For a unit capacitor with $C_{\mu}=2 f F$ and $\sigma\left(\frac{\Delta C}{C_{\mu}}\right)=0.5 \%$, the absolute standard deviation to be measured is only $10 \mathrm{aF}$. If we compare this value to the accuracy of commercially available LCR meters, which can be as low as $10 \%$ for capacitors in femto-Farad range [12], it will be anticipated that the mismatch of such a small capacitor cannot be directly measured by an LCR meter. In addition, parasitic capacitance and measurement variations due to pads, cabling, probing, temperature change, etc., will make such a measurement unreliable. These reasons led to a common notion that the measurement of small capacitors mismatch necessitates the use of custom on-chip circuitry [9], [10].

However, the purpose of mismatch measurement is not to measure the absolute value of the unit capacitor, but rather to quantify the difference between two adjacent and consecutively measured unit capacitors. Thus, the stringent requirement is on the short-term repeatability of the measurement, rather than the measurement accuracy or the longterm repeatability. Fig. 1 shows direct mismatch measurement between two capacitors $\left(C_{A}\right.$ and $\left.C_{B}\right)$, where only $C_{A}$ or $C_{B}$ is connected to the test pads and then to the LCR meter terminals using on-chip switches. This allows fast and consecutive measurement without introducing any change in the measurement environment. Thus, variations due to pads,

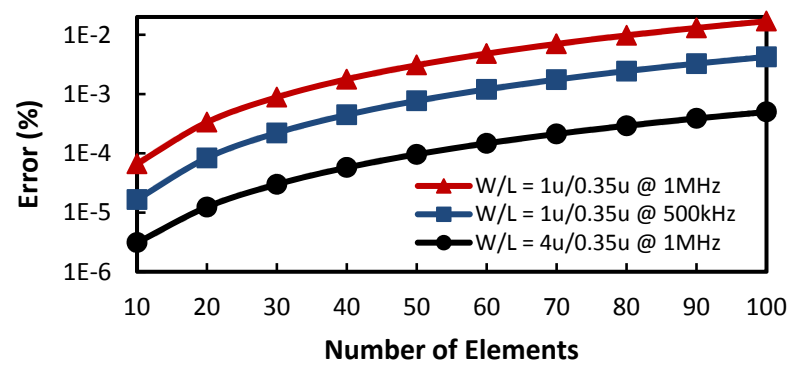

Figure 2. MC circuit simulation results showing percent of error in capacitance mismatch measurement due to switch ON resistance mismatch vs number of unit capacitors connected in parallel for different switch sizes and measurement frequencies.

cabling, probing, temperature change, etc., are effectively eliminated. The LCR meter measures the differential capacitance between the HIGH and LOW terminals, thus the parasitic capacitances $\left(C_{P, H}\right.$ and $\left.C_{P, L}\right)$ do not affect the measurement, where $C_{P, H}$ is connected to the LCR meter signal source (HIGH terminal) and $C_{P, L}$ is connected to a virtual ground (LOW terminal). Moreover, even if the parasitic capacitance affects the absolute measurement due to non-ideal effects, it will not affect the relative mismatch measurement because identical parasitic capacitance appears when measuring $C_{A}$ and $C_{B}$, so both are affected equally. It should be noted that the parasitic capacitance is dominated by the capacitance of the pads, probes, and cables, thus, any mismatch in the parasitic capacitance of the switches is practically negligible. To further improve the measurement accuracy, instead of measuring the mismatch of two unit capacitors only, the mismatch between multiple unit capacitors connected in parallel can be measured, as depicted in Fig. 1, where each of $C_{A}$ and $C_{B}$ is composed of $N$ unit capacitors connected in parallel. In this case, the absolute standard deviation to be measured is given by

$$
\sigma\left(C_{e q}\right)=\sqrt{N} \times \sigma(\Delta C),
$$

where $C_{e q}$ is the equivalent capacitance and $N$ is the number of unit capacitors connected in parallel. Hence, the absolute standard deviation to be measured is increased by a factor of $\sqrt{N}$. This may be even necessary for on-chip mismatch measurement, where 128 unit capacitors were used in [10] in order to produce a single measurement point. The aforementioned measurement is further applied to several pairs of capacitors (similar to $C_{A}$ and $C_{B}$ ) in order to obtain a statistical distribution.

One possible source of error in the measurement configuration shown in Fig. 1 is the mismatch of the switch ON resistance. However, noting that the capacitor-under-test is in the femto-Farad range, the switch $\mathrm{ON}$ resistance is orders of magnitude less than the capacitor reactance for frequencies commonly used in precision LCR meters (typically $\leq 1 M H z)$. Thus, the switch mismatch will have negligible effect on the equivalent impedance. To further verify this, Monte Carlo (MC) circuit simulations were conducted using Spectre mismatch models of the $0.35 \mu \mathrm{m}$ technology which was used to implement the test chip. As shown in Fig. 2, for a pessimistic case of a small switch $(1 \mu \mathrm{m} / 0.35 \mu \mathrm{m})$ and 


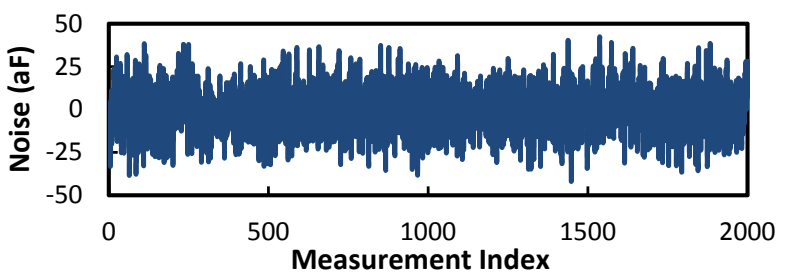

(a)

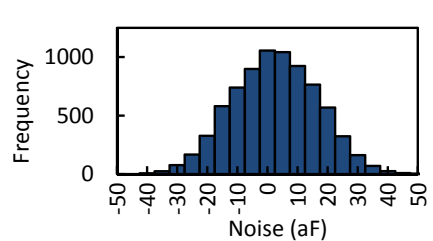

(b)

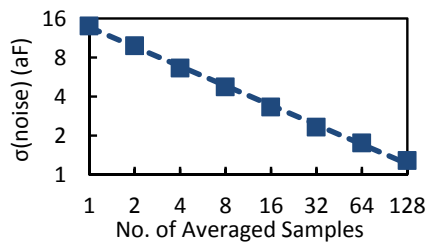

(c)
Figure 3. (a) Short-term repeatability, i.e., measurement noise, of a $14 \mathrm{fF}$ capacitor measurement using commercial LCR meter. (b) Histogram of measurement noise. (c) Noise standard deviation vs number of averaged readings plotted on a log-log scale.

$N=100$, the error is less than $0.02 \%$ at $1 M H z$.

Given the above argument, direct mismatch measurement is only limited by the short-term repeatability error, which is mainly caused by the noise of the instrument electronic circuitry. This short-term repeatability is much better than the instrument accuracy, which allows doing precise direct mismatch measurement. In order to verify this, Fig. 3a shows the short-term repeatability of a $7 \times 2 f F=14 f F$ capacitor measurement performed by a commercial LCR meter (Agilent E4980A), where the standard deviation of the noise is only $14 a F$, which is much better than the instrument accuracy. Moreover, the noise histogram has a normal distribution as shown in Fig. 3b; thus the effect of measurement noise can be further reduced by averaging multiple observations, where the standard deviation is inversely proportional to the square root of the number of averaged readings as shown in Fig. 3c. A standard deviation as small as $5 a F$ can be achieved by averaging 8 samples only, while 131,072 points were averaged in [10] in order to suppress measurement noise.

\section{Capacitive Test Structure}

The test structure used for mismatch measurement in the implemented test chip is a 9 - bit binary weighted programmable capacitor array (PCA) using $2 f F$ poly-insulatorpoly (PIP) unit capacitors. The schematic of the PCA is similar to Fig. 1, where $C_{A}$ and $C_{B}$ are replaced with nine capacitors $\left(C_{0}, C_{1}, \ldots, C_{8}\right)$ with binary weighted number of elements $\left(2^{0}, 2^{1}, \ldots, 2^{8}\right)$. The binary weighted capacitors are arranged in a common centroid scheme as shown in Fig. 4a, where the PCA is split into two halves, where each half is surrounded by dummies and switches are placed in between to simplify routing and minimize systematic mismatch. Fig. 4b shows partial layout of the array where the unit capacitor is formed of the overlap between POLY1 and POLY2 layers. The bottom plates are connected together via METAL1 while top plates are routed in METAL3. The top plate routing is completely shielded using METAL2 layer which is not shown in Fig. 4b

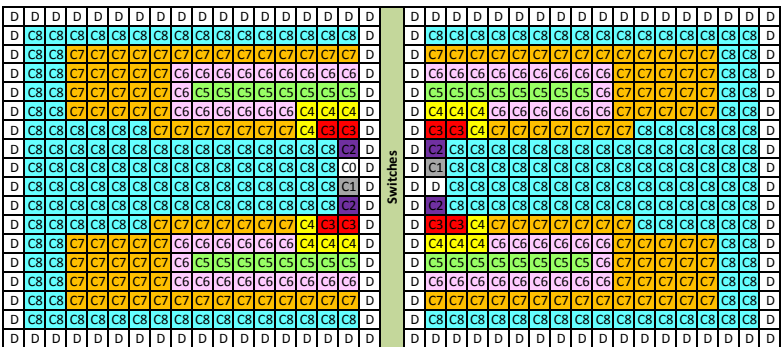

(a)
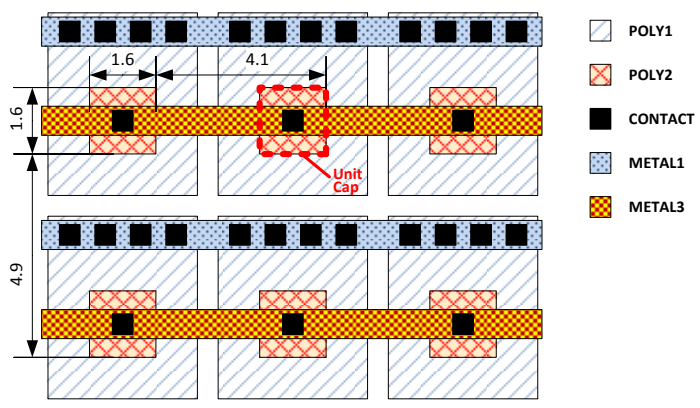

(b)

Figure 4. (a) The PCA is split into two halves in a common-centroid scheme with routing and switches in between. 'D' indicates a dummy capacitor. (b) Partial layout of the PIP capacitor array with dimensions in $\mu \mathrm{m}$. METAL2 shield layer is not shown for the purpose of clarity.

for the purpose of clarity. The unit capacitor area drawn in layout is $1.6 \mu \mathrm{m} \times 1.6 \mu \mathrm{m}$, however measurements indicate an effective area after fabrication of $1.8 \mu \mathrm{m}^{2}$, which is further verified using SEM. The unit capacitors are spaced relatively far from each other due to layout design rules.

When the $i-t h$ bit of the PCA digital input $\left(b_{i}\right)$ switches from zero to one while all other less significant bits $\left(b_{i-1}, b_{i-2}, \ldots, b_{1}\right)$ switch from one to zero, where $b_{1}$ is the least significant bit (LSB), a total of $\left(2^{i}-1\right)$ capacitors are switched. Thus, the variance of the differential non-linearity (DNL) for this $i-t h$ bit transition, which we will refer to as a major transition, will be given by

$$
\sigma^{2}\left(D N L_{i}\right)=\left(2^{i}-1\right) \sigma^{2}\left(\frac{\Delta C}{C_{\mu}}\right),
$$

Thus, the relative standard deviation of the unit capacitor is given by

$$
\sigma\left(\frac{\Delta C}{C_{\mu}}\right)=\sigma\left(\frac{D N L_{i}}{\sqrt{2^{i}-1}}\right) .
$$

Hence, the distribution of measured DNL from several PCAs can be used to estimate the distribution of unit capacitor mismatch [5].

In order to investigate the effect of LCR meter measurement noise on mismatch calculation using the aforementioned technique, a MATLAB MC simulation was conducted. Ten binary weighted PCAs with a unit capacitor of $2 f F$ mean value and $0.5 \%$ relative standard deviation were simulated, which is the same number of experimentally measured PCAs as will be shown in Sec. IV. The DNL of the simulated PCAs was computed in three cases; 1) Zero noise added, 2) $5 a F$ rms noise, which is the measured rms noise when 


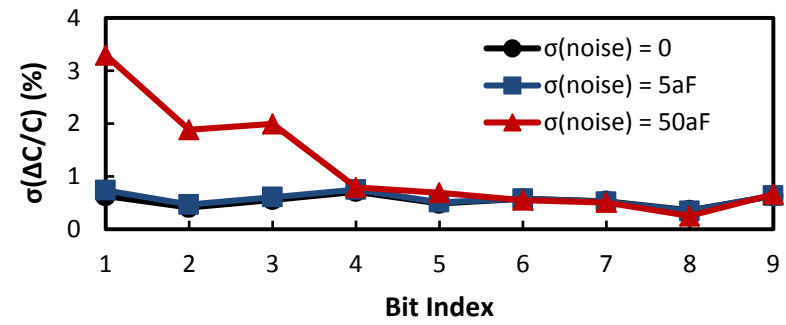

(a)

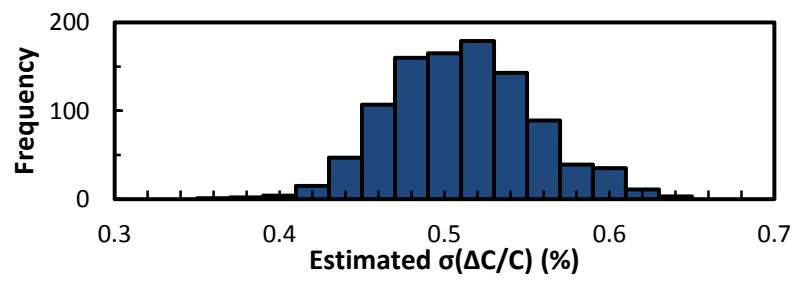

(b)

Figure 5. (a) MATLAB MC simulation showing the estimated unit capacitor relative standard deviation vs bit index of PCA digital input for $\sigma($ noise $)=0,5 a F$, and $50 a F$. (b) Statistical distribution of the relative standard deviation estimated from 10 PCAs.

8 samples are averaged, and 3) a ten times exaggerated rms noise of $50 a \mathrm{~F}$. The relative standard deviation of the unit capacitor was then calculated using (4), where $i=1,2, \ldots, 9$ as shown in Fig. 5a, where for the exaggerated case of $50 a \mathrm{~F}$ rms noise, the noise is dominant for the LSBs; however, its effect is less severe for the most significant bits (MSBs), as expected from (2). On the other hand, for the case of $5 a F$ rms noise, the calculated relative standard deviation almost matches the zero noise case, which shows that direct mismatch measurement is possible using the proposed structure while averaging 8 readings only. To further investigate the error in the estimated $\sigma\left(\frac{\Delta C}{C_{\mu}}\right)$, an additional MC simulation was conducted where the previous simulation was repeated 1000 times and the resulting distribution is plotted in Fig. 5b, where the estimated $\sigma\left(\frac{\Delta C}{C_{\mu}}\right)$ has an rms error of $0.04 \%$.

\section{Results And Discussion}

The PIP 9-bit PCA structures were fabricated in a low-cost $0.35 \mu \mathrm{m}$ CMOS process. Fig. 6a shows a die microphotograph of one fabricated PCA occupying an area of $230 \times 90 \mu \mathrm{m}^{2}$ and Fig. 6b shows an SEM cross-sectional photo of the fabricated PCA. The fabricated chip is packaged and mounted on a test board. The control of on-chip digital switches is done via a 4-wire serial peripheral interface (SPI) bus and a LabVIEW interface is designed for testing automation and data acquisition from an E4980A LCR meter.

Fig. 7 shows the unit capacitor relative standard deviation calculated from measured DNL using (4) at each major bit transition of the 9 - bit PCA. A total of 10 PCAs were characterized yielding a total of 90 mismatch measurement points. The estimated average relative standard deviation is $0.43 \%$, where the rms error is in the order of $0.04 \%$ as estimated in Sec. III. This result is further compared to mismatch measurement of large PIP capacitors that are implemented in

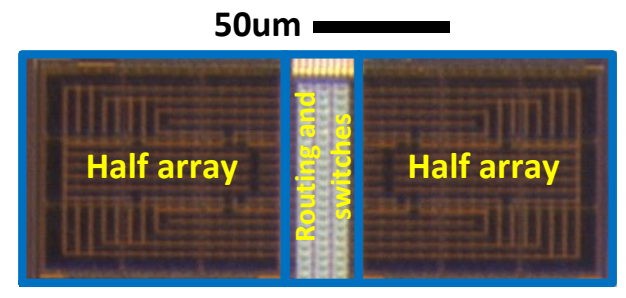

(a)

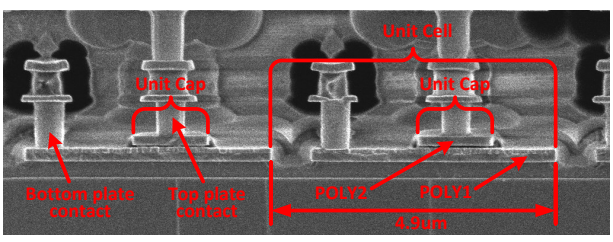

(b)

Figure 6. (a) Microphotograph of the fabricated PCA. (b) SEM cross-sectional photo of the fabricated PCA showing two PIP unit capacitors.

the same process. Fig. 8 shows Pelgrom's model [7] fitted to measured data of large capacitors and extrapolated to small capacitor region. It can be clearly noticed that the measured mismatch for the $2 f F$ unit capacitor is higher than what is estimated from Pelgrom's model. This can be explained by noting that the commonly used form of Pelgrom's model is a simplified form of the full model which only considers arearelated effects. However, a more general capacitor mismatch model that considers both area and edge random effects is given by [7], [11]

$$
\sigma\left(\frac{\Delta C}{C_{\mu}}\right)=\sqrt{\frac{K_{A}^{2}}{A}+\frac{K_{E}^{2}}{A^{\frac{3}{2}}},}
$$

where $A$ is the capacitor top-view area, $K_{A}$ is the area dependence coefficient and $K_{E}$ is the edge dependence coefficient. For large area devices, area effects will dominate, and (5) will reduce to Pelgrom's $1 / \sqrt{A}$ dependence [7]. However, for small areas edge effects will start to dominate, and the mismatch will increase at a higher rate as the area shrinks because it will be inversely proportional to $A^{\frac{3}{4}}$ instead of $A^{\frac{1}{2}}$. The cross-over value between area and edge effects will vary from one process to another and from one capacitor type to another [7], [11]. Although measurement data to estimate the cross-over value is not usually available, the prudent designer should expect the mismatch of very small capacitors to be as large as twice the mismatch estimated from extrapolated models based on large capacitors. It should be noted that mismatch models included in process design kits are usually based on measurements of large capacitor mismatch only.

Table I compares the reported mismatch measurement with mismatch measurement of small metal-oxide-metal (MOM) capacitors [5], [10]. It is noteworthy that although the measured $2 f F$ PIP capacitor mismatch is higher than what is expected from Pelgrom's model, it is still better than the mismatch of MOM capacitor implemented in an advanced $32 \mathrm{~nm}$ CMOS process [10]. For a fair comparison, we need to compare two capacitors of the same nominal value. The mismatch of the capacitors reported in [10] follows Pelgrom's 
Table I

COMPARISON OF MEASURED MATCHING PROPERTIES WITH RECENTLY PUBLISHED WORK.

\begin{tabular}{|c|c|c|c|c|}
\hline & JSSC & \multicolumn{2}{|c|}{ TCAS-I } & This \\
& $2011[5]$ & \multicolumn{2}{|c|}{$2014[10]$} & Work \\
\hline Capacitor Type & MOM & MOM & MOM & PIP \\
\hline Field Direction & Lateral & Lateral & Lateral & Vertical \\
\hline Technology $(n m)$ & 90 & 32 & 32 & 350 \\
\hline Unit Cap Area $\left(\mu m^{2}\right)$ & 0.74 & 1.8 & 4.8 & 1.8 \\
\hline$C_{\mu}(f F)$ & 0.5 & 0.45 & 1.2 & 2 \\
\hline$\sigma\left(\frac{\Delta C}{C_{\mu}}\right)$ & $<1 \%$ & $1.2 \%$ & $0.8 \%$ & $0.43 \%$ \\
\hline
\end{tabular}

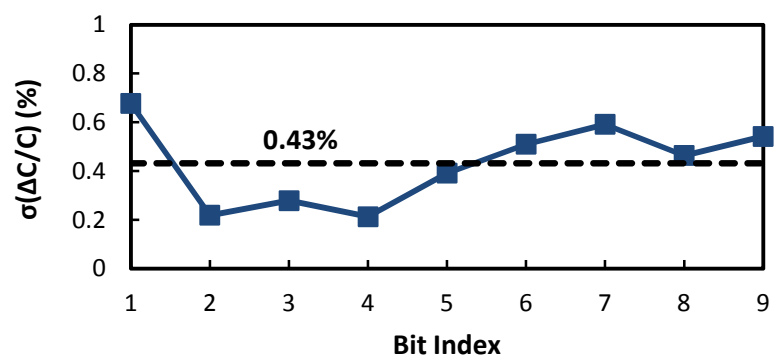

Figure 7. Relative standard deviation of unit capacitor calculated from measured data of 10 PCAs vs bit index of the PCA digital input.

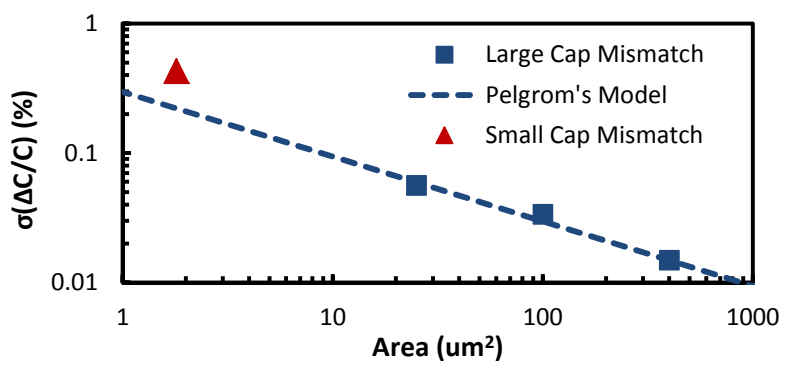

Figure 8. Measured mismatch data for small and large unit capacitors vs capacitor area overlaid on models of area dominated mismatch (Pelgrom's Model) and mismatch due to random edge variations plotted on a log-log scale.

model; thus we can estimate the mismatch of a $2 f F$ MOM capacitor to be $0.6 \%$, which is higher than the mismatch of the reported $2 f F$ PIP capacitor. Two metal layers are stacked in [5] which results in a smaller area compared to [10], where a single layer is used. The unit capacitor area, which is a main factor affecting mismatch, of the MOM capacitors is larger than a PIP capacitor of the same value. However, it should be noted that when considering routing and design rules, the unit cell area of a PIP capacitor in $0.35 \mu \mathrm{m}$ process will be larger.

In [13] and [10], it was suggested that capacitors that have lateral fields, e.g., MOM capacitors, should have better matching properties compared to vertical field capacitors, because lateral capacitors are primarily defined by lithography which is quite accurate in a modern CMOS process. However, as can be noted in Table I, although the lithography feature size is better by more than one order of magnitude, lateral capacitor did not show better matching compared to vertical capacitor. In addition, it should be noted that in both lateral and vertical capacitors, the capacitance is determined by both lateral and vertical dimensions altogether, e.g., in MOM the capacitance is affected by metal thickness which is a vertical dimension. This shows that lateral capacitors do not necessarily match better, especially when the fabrication process and the capacitor type are different.

\section{CONCLUSION}

Direct mismatch measurement of small capacitors is feasible and can be used for capacitors of different types. The mismatch of a $2 f F$ PIP capacitor was directly measured using simple test structure that can be automated and ported. Measurements show that small capacitors will suffer from increased mismatch compared to the extrapolation of Pelgrom's model that was extracted from mismatch of large capacitors. Small PIP capacitors implemented in a low-cost $0.35 \mu \mathrm{m}$ process show better mismatch compared to MOM capacitors implemented in a deep-sub-micron $32 \mathrm{~nm}$ process, indicating that smaller feature size and lateral field capacitors do not necessarily match better, especially when comparing capacitors of different types and from different technologies.

\section{ACKNOWLEDGMENT}

The authors would like to thank Prof. Boon S. Ooi for facilitating the SEM imaging of the structures.

\section{REFERENCES}

[1] H. Lee, S. Park, C. Lim, and C. Kim, "A 100-nW 9.1-ENOB 20kS/s SAR ADC for Portable Pulse Oximeter," Circuits and Systems II: Express Briefs, IEEE Transactions on, vol. 62, no. 4, pp. 357-361, April 2015.

[2] J.-H. Tsai, H.-H. Wang, Y.-J. Chen, Y.-H. Wei, Y.-H. Kao, Y.-C. Yen, P.-C. Huang, M.-H. Shen, and H. Chen, "A 1-V-0.6-V 9-b 1.5MS/s Reference-Free Charge-Sharing SAR ADC for Wireless-Powered Implantable Telemetry," Circuits and Systems II: Express Briefs, IEEE Transactions on, vol. 61, no. 11, pp. 825-829, Nov 2014.

[3] H. Omran, M. Arsalan, and K. N. Salama, "A robust parasitic-insensitive successive approximation capacitance-to-digital converter," in Custom Integrated Circuits Conference (CICC), 2014 IEEE Proceedings of the, Sept 2014.

[4] - "An integrated energy-efficient capacitive sensor digital interface circuit," Sensors and Actuators A: Physical, vol. 216, pp. 43 - 51, 2014.

[5] P. Harpe, C. Zhou, Y. Bi, N. van der Meijs, X. Wang, K. Philips, G. Dolmans, and H. De Groot, "A 26 uW 8 bit $10 \mathrm{MS} / \mathrm{s}$ Asynchronous SAR ADC for Low Energy Radios," Solid-State Circuits, IEEE Journal of, vol. 46, no. 7, pp. 1585-1595, 2011.

[6] M. Saberi, R. Lotfi, K. Mafinezhad, and W. Serdijn, "Analysis of Power Consumption and Linearity in Capacitive Digital-to-Analog Converters Used in Successive Approximation ADCs," Circuits and Systems I: Regular Papers, IEEE Transactions on, vol. 58, no. 8, pp. 1736-1748, 2011.

[7] M. Pelgrom, A. C. J. Duinmaijer, and A. Welbers, "Matching properties of MOS transistors," Solid-State Circuits, IEEE Journal of, vol. 24, no. 5, pp. 1433-1439, 1989.

[8] H. Omran, M. Arsalan, and K. N. Salama, "7.9 pJ/Step EnergyEfficient Multi-Slope 13-bit Capacitance-to-Digital Converter," Circuits and Systems II: Express Briefs, IEEE Transactions on, vol. 61, no. 8, pp. 589-593, Aug 2014.

[9] A. Verma and B. Razavi, "Frequency-Based Measurement of Mismatches Between Small Capacitors," in Custom Integrated Circuits Conference, 2006. CICC '06. IEEE, Sept 2006, pp. 481-484.

[10] V. Tripathi and B. Murmann, "Mismatch Characterization of Small Metal Fringe Capacitors," Circuits and Systems I: Regular Papers, IEEE Transactions on, vol. 61, no. 8, pp. 2236-2242, Aug 2014.

[11] J.-B. Shyu, G. Temes, and K. Yao, "Random errors in MOS capacitors," Solid-State Circuits, IEEE Journal of, vol. 17, no. 6, pp. 1070-1076, Dec 1982.

[12] E4980A Precision LCR Meter Data Sheet. Agilent Technologies, 2011.

[13] R. Aparicio and A. Hajimiri, "Capacity limits and matching properties of integrated capacitors," Solid-State Circuits, IEEE Journal of, vol. 37, no. 3, pp. 384-393, Mar 2002. 